\title{
Forcing Transmembrane Voltages to Decrease Slowly: A Temporal Regularization for ECG Imaging
}

\author{
Steffen Schuler ${ }^{1}$, Axel Loewe $^{1}$ and Olaf Dössel ${ }^{1}$ \\ ${ }^{1}$ Institute of Biomedical Engineering, Karlsruhe Institute of Technology (KIT), Karlsruhe, Germany
}

\begin{abstract}
ECG imaging aims to reconstruct the cardiac electrical activity from non-invasive measurements of body surface potentials (BSP) by finding unique and physiologically meaningful solutions to the inverse problem of electrocardiography. This can be accomplished using regularization, which reduces the space of admissible solutions by demanding solution properties that are already known beforehand. Messnarz et. al. proposed a regularization scheme that requires transmembrane voltages (TMV) to not decrease over time. We suggest a generalization of this method that forces TMVs to decrease only slowly and as a result can also be applied to irregular cardiac activity. We first develop the method using a simplified spherical geometry and then show its benefit for imaging fibrillatory activity on a realistic geometry of the atria.
\end{abstract}

\section{Introduction}

The transfer of electrical potentials from the heart to the body surface represents an attenuation and spatial smoothing. Reversing this process leads to an amplification of measurement noise and is generally not unique, as many cardiac source patterns with high spatial frequency can result in smoothed BSP patterns that differ only by a magnitude smaller than the measurement noise. In a more mathematical way, this can be seen from a singular value decomposition (SVD) of the lead field matrix, which shows that increasingly high frequency source basis patterns are associated with declining singular values. A unique solution can be obtained by truncating the SVD or explicitly forcing the source pattern to be of low spatial frequency. This motivates the use of Tikhonov regularization, especially in its second order form that minimizes the Laplacian of the solution. While this approach is sensible from a purely electrodynamic point of view, it can be questioned from a physiological perspective. Spatial source patterns resulting from the physiological spread of cardiac excitation waves are characterized by a sharp edge caused by depolarization and a less steep gradient caused by repolarization. Classi- cal regularization methods smear the depolarization edge in space - and as a consequence often also in time. Total variation regularization can be used to promote sharp edges by assuming source values to be piecewise constant in space. However, this assumption is violated for transmembrane voltages during repolarization, which may also impede the reconstruction of other node's depolarization. In the following, we want to explore a new idea for regularization in the temporal domain that makes use of the stereotypical time course of action potentials and might allow for a less severe spatial regularization.

\section{Methods}

\subsection{Geometries and excitation patterns}

In this work, we use two different sets of geometries (Fig. 1). A spherical test geometry is used to study the effect of different regularization configurations under
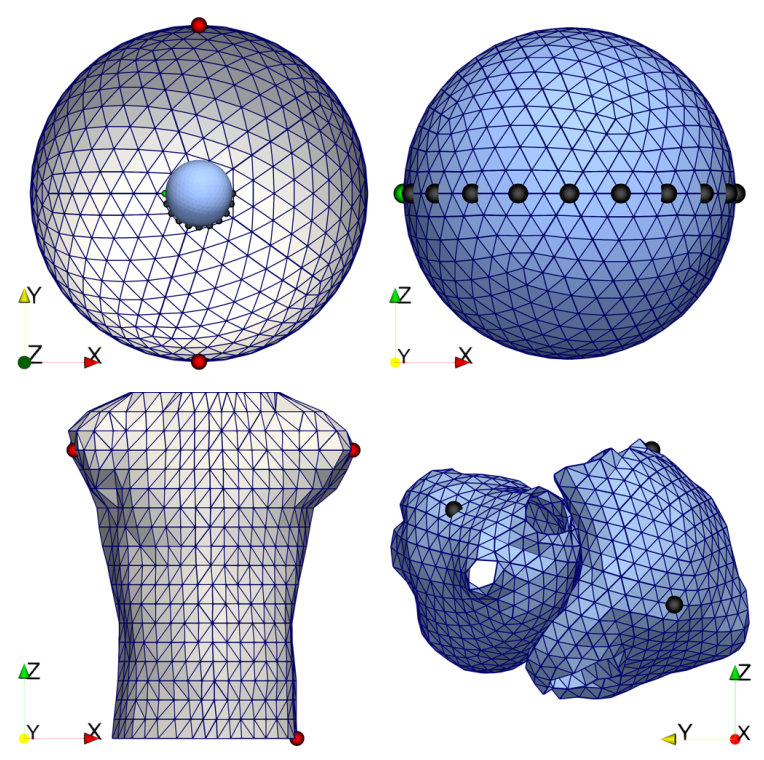

Figure 1. Top: Spherical test geometry. Bottom: Realistic torso-atria geometry. Green dot: stimulus location. Red dots: Reference electrodes. Black dots: Evaluation nodes. 
idealized conditions. It consists of three concentrically aligned icosphere surfaces with 642 nodes each. The outmost surface has a diameter of $25 \mathrm{~cm}$ and represents the torso surface. The other two surfaces have a diameter of 5 and $4.6 \mathrm{~cm}$ and represent the epi- and endocardial surfaces bounding the myocardium. With a diameter of $5 \mathrm{~cm}$ and a wall thickness of $2 \mathrm{~mm}$, the geometry best resembles the left atrium. Monodomain simulations of a focal activity were performed on a finer tetrahedral mesh of the spherical myocardium and transferred onto the surface mesh. We used the atrial cell model by Courtemanche et al. [1]. Homogeneous and isotropic monodomain conductivities were used and adapted to obtain a conduction velocity of $800 \mathrm{~mm} / \mathrm{s}$. The stimulus location is marked by a green dot in Fig. 1. The black dots mark 10 evaluation nodes placed equidistantly along the equator parallel to the direction of excitation propagation.

A more realistic geometry of the torso and the atria is later used to demonstrate the new regularization method in an application to irregular atrial activity. The geometry is the same as in [2], but the number of atrial nodes has been reduced to 1696 in order to speed up computations. The "simple atrial fibrillation" simulation from [3] is chosen as activity to be reconstructed, which includes rotational activity on the right atrium repeating at $7.3 \mathrm{~Hz}$, while the left atrium is activated at $4.7 \mathrm{~Hz}$.

\subsection{Forward calculations}

We assume equal intra-to-extracellular anisotropy ratios and thus the BSPs can be calculated from transmembrane voltages on the surface bounding the myocardium [4]. Furthermore, the torso is assumed isotropic and thus the lead field matrix A can be obtained using the boundary element method. The forward model then is:

$$
\mathbf{b}(t)=\mathbf{A x}(t)+\varepsilon(t)
$$

where $\mathbf{b}(t)$ are the BSPs, $\mathbf{x}(t)$ the surface TMVs and $\varepsilon(t)$ white gaussian noise for all nodes at a time $t$. All nodes of the torso models are used as electrodes. It is assumed that each electrode is affected by the same absolute noise power, which is set to the average signal power of all nodes divided by the signal-to-noise ratio (SNR). We use an SNR of $0 \mathrm{~dB}$ for the spherical geometry and an SNR of $20 \mathrm{~dB}$ for the realistic geometry. BSPs are not filtered.

\subsection{Inverse calculations}

For solving the inverse problem, we assume perfect knowledge of $\mathbf{A}$ and thus neglect errors due to imperfect geometries and conductivities. The epi-endo projection from [2] is applied for both geometries to resolve ambiguities between sources on the epi- and endocardial surfaces by reducing the source space to effectively one layer.

\subsubsection{New temporal regularization}

The regularization method by Messnarz et. al. [5] reduces the solution space by restricting TMVs to be nondecreasing over time. It works well, if for every node only one depolarization and ideally only the subsequent plateau phase lies within the time window to be reconstructed. However, it cannot be used to simultaneously reconstruct multiple successive beats or even irregular activity arising during arrhythmias. In order to overcome this problem, we suggest to slightly loosen the restriction of non-decreasing TMVs by imposing a small negative lower bound on the temporal derivative instead. Additionally, we add a zero-order constraint that pulls TMVs to their lower bound. From a physiological view, this can be seen as a force to bring the system back to its resting state. The regularized least-squares problem can be written as follows:

$$
\begin{aligned}
\mathbf{x}(t)=\arg \min _{\mathbf{x}(t)}\{ & \|\mathbf{A} \mathbf{x}(t)-\mathbf{b}(t)\|_{2}^{2} \\
& \left.+\lambda\|\mathbf{L} \mathbf{x}(t)\|_{2}^{2}+\eta\left\|\mathbf{x}(t)-V_{\min }\right\|_{2}^{2}\right\} \\
\text { s.t. } \quad & V_{\max } \geq \mathbf{x}(t) \geq V_{\min } \\
& \mathbf{x}(t+1)-\mathbf{x}(t) \geq s_{\min }
\end{aligned}
$$

$\mathbf{L}$ is an approximation of the surface Laplacian operator. $V_{\min }$ and $V_{\max }$ are lower and upper bounds of the TMV, respectively and $s_{\min }$ is a lower bound of the TMV slope. This quadratic problem with linear constraints is solved simultaneously for 200 consecutive time steps à $\Delta t=2 \mathrm{~ms}$. We used the freely available software package CVX [6] in combination with MOSEK's conic interior point optimizer. The focal activity for the spherical geometry comprised only $400 \mathrm{~ms}$ and could thus be solved at once. The irregular activity for the realistic atrial geometry however spans $4 \mathrm{~s}$. Therefore we split the whole time span in 20 segments à 200 time steps with an overlap of $50 \%$. The solution was then joined together from the 100 central time steps of each segment.

Three different configurations of (1), shown in Table 1, are evaluated with the spherical geometry: temporal slowdecreasing (TSD), TSD without Laplacian and temporal non-decreasing (TND), which corresponds to Messnarz's method. For the realistic atrial geometry, only TSD is used.

Table 1. Regularization configurations for (1).

\begin{tabular}{|l|c|c|c|c|c|}
\cline { 2 - 6 } \multicolumn{1}{c|}{} & $\lambda$ & $\eta$ & $\begin{array}{c}V_{\min } \\
(\mathrm{mV})\end{array}$ & $\begin{array}{c}V_{\max } \\
(\mathrm{mV})\end{array}$ & $\begin{array}{c}s_{\min } / \Delta t \\
(\mathrm{mV} / \mathrm{ms})\end{array}$ \\
\hline $\begin{array}{l}\text { TSD } \\
\text { (default) }\end{array}$ & $\neq 0$ & $\neq 0$ & -80 & $\infty$ & -0.5 \\
\hline $\begin{array}{l}\text { TSD w/o } \\
\text { Laplacian }\end{array}$ & $\mathbf{0}$ & $\neq 0$ & -80 & $\infty$ & -0.5 \\
\hline TND & $\neq 0$ & $\mathbf{0}$ & -80 & $\mathbf{0}$ & $\mathbf{0}$ \\
\hline
\end{tabular}


Applying an a posteriori parameter choice method like the L-curve method requires to compute solutions for many different parameter values. As solving problem (1) is computationally very expensive, we manually tuned the regularization parameters in steps of whole decades until a visually meaningful solution was obtained.

\subsubsection{Tikhonov regularization}

Reconstructions using the new method are compared with a Tikhonov regularization (Tikh) using zero- and second-order terms [7]:

$$
\mathbf{X}=\arg \min _{\mathbf{X}}\left\{\|\mathbf{A} \mathbf{X}-\mathbf{B}\|_{F}^{2}+\lambda\|\mathbf{L X}\|_{F}^{2}+\eta\|\mathbf{X}\|_{F}^{2}\right\}
$$

$\mathbf{B}$ and $\mathbf{X}$ contain all time steps along the column dimension. As Tikhonov regularization is applied to all time steps simultaneously, the regularization parameters $\lambda$ and $\eta$ are constant over time. For fibrillatory activity, it was found that constant parameters do not impair the solution quality compared to instantaneous ones [2]. In this work, the optimal Tikhonov parameters were obtained by maximizing the temporal mean of spatial TMV correlation with the ground truth using downhill simplex optimization.

\subsection{Post-processing and metrics}

Similarity between true and reconstructed TMVs is measured using the Pearson correlation coefficient (CC) in two versions: The temporal $\mathrm{CC}$ is computed separately for each node using all time steps while the spatial CC treats nodes and time steps the opposite way. To quantify similarity of high frequency components of spatial TMV distributions, we calculate the spatial CC for the gradient norm of TMVs as well. Local activation times (LAT) are extracted and compared as described in [2]. In short, the peaks of a spatio-temporal derivative signal [8] are detected and absolute errors between only true positive detections are calculated together with the false negative rate (FNR) and the false positive rate (FPR).

\section{Results}

\subsection{Spherical geometry}

Results for the configurations given in Table 1 and for Tikhonov regularization are depicted in Fig. 2. It can be seen that TSD without Laplacian (B) is able to recover the steep depolarization gradient in both space and time. Tikh with optimal parameters (E) led to spatially strongly smoothed TMVs and a less steep temporal upstroke. TSD with a Laplacian term weighted by the same $\lambda$ as for Tikh led to a less severe smoothing in space and largely retained temporal steepness. LAT errors were best for TND and second best for TSD. Therefore we decided to use TSD for the following atrial fibrillation reconstructions.
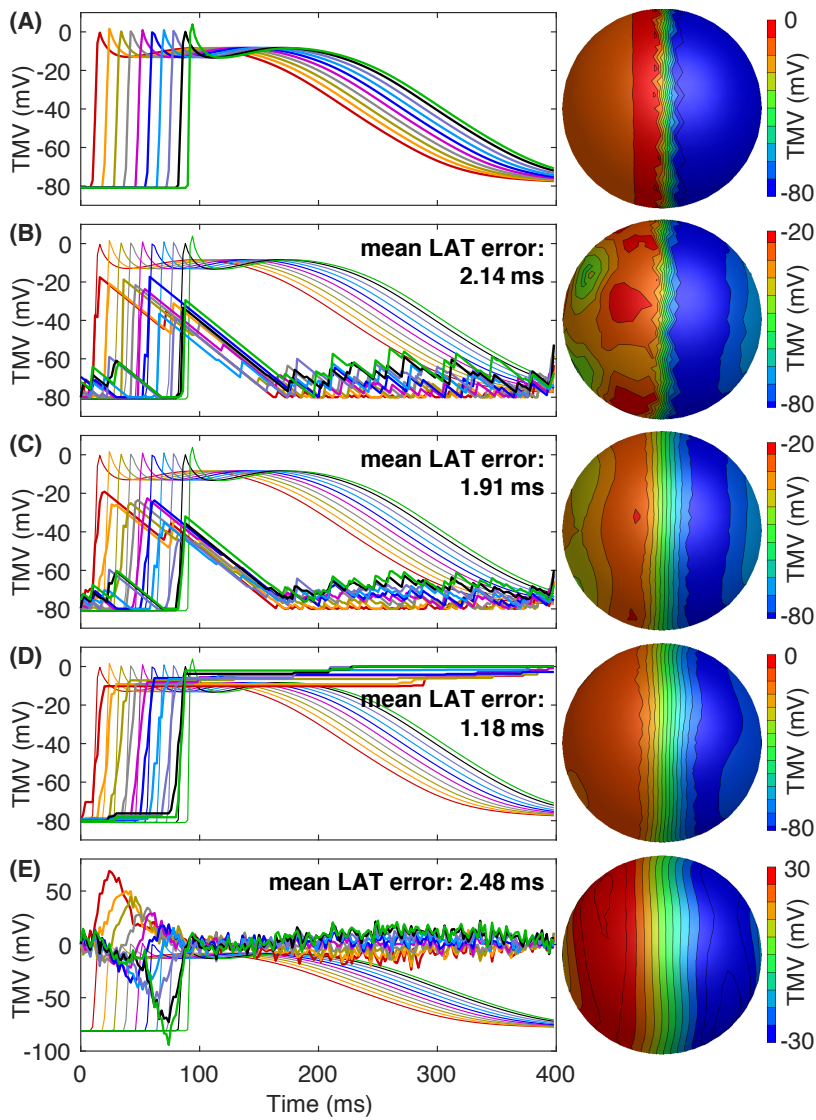

Figure 2. Comparison of reconstructions for the spherical geometry. (A) Ground truth. (B) TSD without Laplacian. (C) TSD. (D) TND. (E) Tikh. Left: TMV time courses at the 10 evaluation nodes marked in Fig. 1 (the truth is added in each graph). Right: Spatial TMV patterns at $50 \mathrm{~ms}$.

\subsection{Realistic atrial geometry}

Fig. 3 shows that TSD is also able to recover spatial details for the atrial fibrillation case that are lost with Tikh.

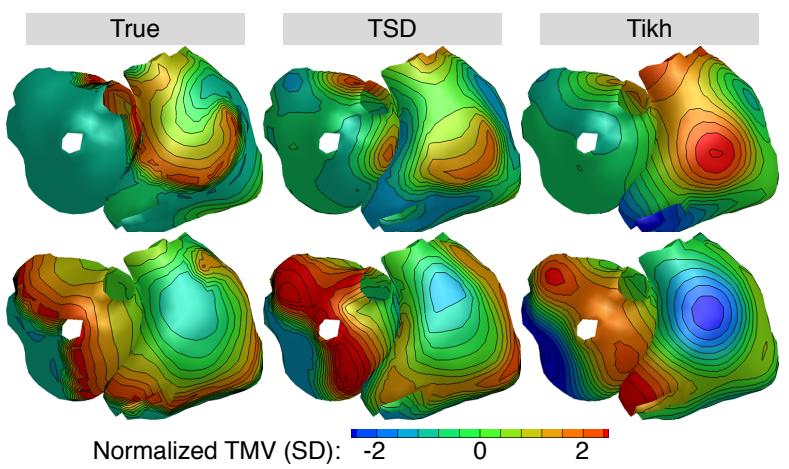

Figure 3. TMV patterns for two time steps $30 \mathrm{~ms}$ apart. 
This is also reflected by the metrics in Fig. 4. Although the spatial CC of TMVs is smaller for TSD than for Tikh that was parameterized to maximize this measure, the spatial CC of the TMV gradient norm is markedly larger for TSD.
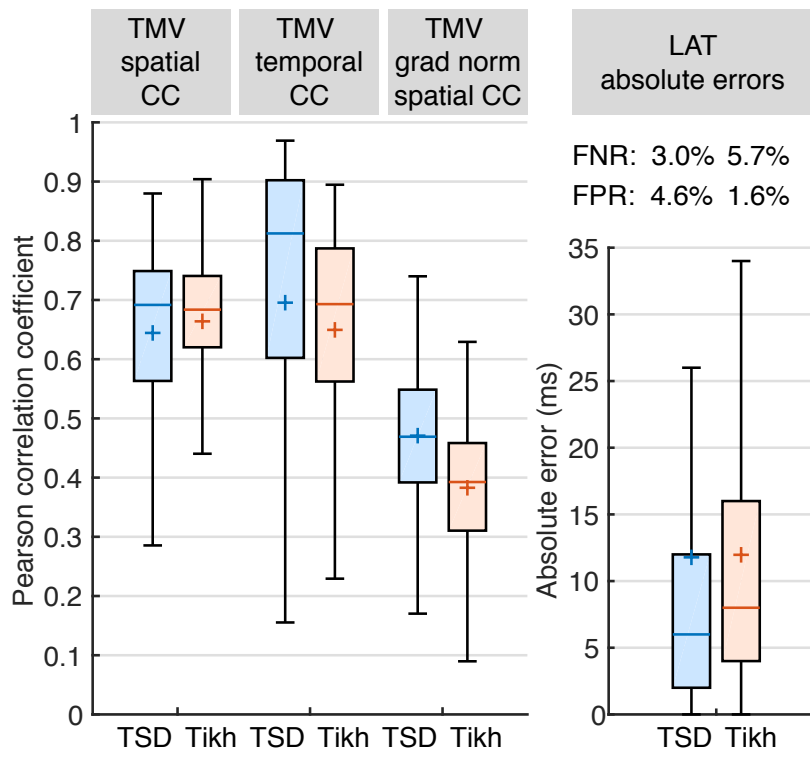

FNR: $3.0 \%$ 5.7\%

FPR: $4.6 \% 1.6 \%$

Figure 4. Metrics for atrial reconstructions.
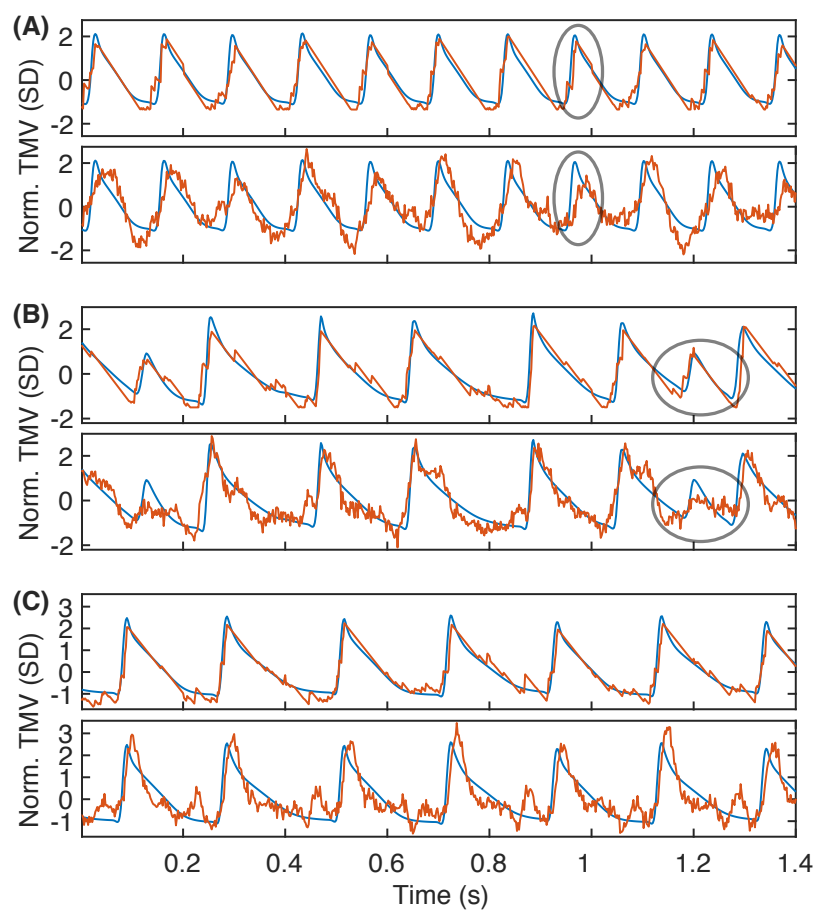

Figure 5. Time courses of reconstructed (red) and true (blue) TMVs for the three atrial evaluation nodes marked in Fig. 1. (A) RA lateral wall. (B) RA appendage. (C) LA posterior wall. The first diagram in each group shows the results for TSD and the second for Tikh. Circles indicate regions, where Tikhonov led to a flattened time course.
The time courses in Fig. 5 reveal that TSD is able to reconstruct the sharp depolarization edges even in cases, where Tikhonov led to flattened TMVs (encircled regions).

\section{Discussion and conclusion}

The good correspondence between time courses reconstructed with TSD and true time courses for atrial fibrillation, especially during repolarisation, might in part be attributed to the triangular shape of action potentials. Experiments with the spherical geometry and the Courtemanche cell model showed that the linear decay does not necessarily correspond to true repolarization. A way to efficiently determine the optimal regularization parameters for TSD still has to be developed. In conlusion, the new temporal regularization forcing TMVs to decrease slowly can be applied to irregular cardiac activity and helps to recover spatial as well as temporal details in inverse reconstructions.

\section{References}

[1] Courtemanche M, Ramirez RJ, Nattel S. Ionic mechanisms underlying human atrial action potential properties: Insights from a mathematical model. Am J Physiol 1 1998; 275:H301-H321.

[2] Schuler S, Potyagaylo D, Dössel O. ECG imaging of simulated atrial fibrillation: Imposing epi-endocardial similarity facilitates the reconstruction of transmembrane voltages. In Computing in Cardiology, volume 44. 2017; .

[3] Figuera C, Suárez-Gutiérrez V, Hernández-Romero I, Rodrigo M, Liberos A, Atienza F, Guillem MS, Barquero-Pérez Ó, Climent AM, Alonso-Atienza F. Regularization techniques for ecg imaging during atrial fibrillation: A computational study. Frontiers in Physiology 2016;7:466.

[4] Geselowitz DB. Description of cardiac sources in anisotropic cardiac muscle: application of bidomain model. Journal of Electrocardiology 1992;25:65-67.

[5] Messnarz B, Tilg B, Modre R, Fischer G, Hanser F. A new spatiotemporal regularization approach for reconstruction of cardiac transmembrane potential patterns. IEEE Transactions on Biomedical Engineering 1 2004;51(2):273-281.

[6] Grant M, Boyd S. CVX: Matlab software for disciplined convex programming, version 2.1. http://cvxr.com/ cvx, March 2014.

[7] Brooks DH, Ahmad GF, MacLeod RS, Maratos GM. Inverse electrocardiography by simultaneous imposition of multiple constraints. IEEE Trans Biomed Eng 1999;46(1):3-18.

[8] Erem B, Brooks DH, van Dam PM, Stinstra JG, MacLeod RS. Spatiotemporal estimation of activation times of fractionated ecgs on complex heart surfaces. In Engineering in Medicine and Biology Society. IEEE, 2011; 5884-5887.

Address for correspondence:

Steffen Schuler, publications@kit.edu

Fritz-Haber-Weg 1, 76131 Karlsruhe, Germany 